\title{
Research on Dynamic Characteristics of Serial-parallel Combination Joint with Flexible-Cable
}

\author{
Lijin Fang ${ }^{1, a}$, Yongle Wei, ${ }^{1, b}$, Guanghong Tao ${ }^{1, c}$
}

${ }^{1}$ School of Mechanical Engineering and Automation, Northeastern University, Shenyang China

aljfang@me.neu.edu.cn, bweiyongle@sohu.com, 'taoguanghong878@126.com

Key words: Parallel driven, Flexible-cable driven, Inspection Robot

\begin{abstract}
In order to design the humanoid dual-arms high voltage transmission line inspection robot, the robot model is given and the working mechanism of the robot crossing obstacles is introduced, and the motion trajectory of the robot when it is moving is analyzed. The dynamic equations of the serial driven and the serial-parallel driven robotic arm are presented based on Lagrange equation and the dynamic characteristics of the wrist and elbow joint are simulated. The numerical simulation result of the serial driven robotic arm shows that the elbow joint has great torque when the robot moves horizontally and vertically. However, the numerical simulation result of the serial-parallel driven robotic arm shows that the elbow joint torque reduces greatly comparing with the serial driven arm. By analyzing the results of the simulation experiment, it can be seen that this serial-parallel combination driven joint with flexible-cable can reduce the elbow torque greatly and improve the load capacity of robotic arm comparing with the serial driven joint, which can solve the problem that the torque of serial driven joint in dual-arms inspection robot is too high.
\end{abstract}

\section{Introduction}

Robotic driven system is one of the important parts of the robotic technology study. The characteristics of the parallel driven robot are high stiffness, precision and speed, but the workspace and flexibility of the parallel driven robot are poor [1]. The traditional serial driven robot has smaller load capacity, and sometimes it can't meet the requirements of practical work, but the serial-parallel driven redundant robot can effectively solve this problem [2]. The serial-parallel driven mode has some advantages such as compact structure, large output torque and large workspace, so many scholars are interested in the serial-parallel driven mode[2 $\sim 4]$. The kinematics of the serial-parallel redundancy driven robot was studied in the reference [2], and the further research was carried by classifying the obstacles as typical convex obstacles and concave obstacle. A kind of the selfish reconstruction more-module mobile robot was designed based on the series-parallel equivalent ball hinge institutions in the reference [3], and the validity and availability of the design was proved. A new kind of the series-parallel Chinese medicine massage robot based on 2-DOF parallel mechanism was presented in the reference [4], which could meet the requirements of Chinese medicine massage. The 2-DOF parallel mechanism of this robot was analyzed, and the positive and negative solutions of the kinematics were found out.

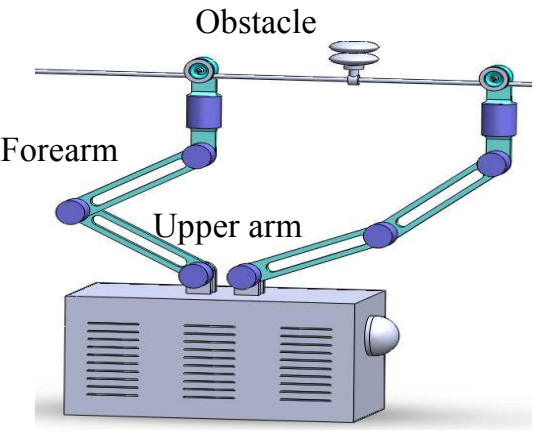

Fig. 1. Model of the dual-arms inspection robot

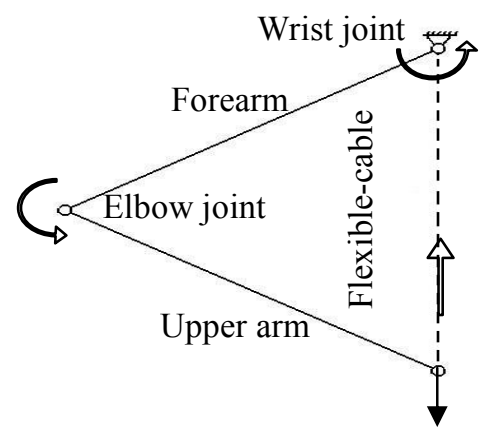

Fig. 2. Serial-parallel driven joint with flexible-cable 
With designing the humanoid dual-arms high voltage transmission line inspection robot shown in Fig.1, a kind of new-type serial-parallel combination driven joint that combine parallel flexible-cable driven mode in serial joint is designed, which is shown in Fig.2. The characteristics of the flexible-cable driven mode such as small inertial, high load capacity are used in this joint. The dynamic equation of the serial-parallel combination driven robotic arm is presented based on Lagrange equation and the dynamic characteristics of the wrist and elbow joints are analyzed.

\section{Working Mechanism of the Robotic Arm}

In order to insure the reliability of the overhead high-voltage transmission Lines and improve the efficiency of line inspection, developing the inspection robot has become one of the hot spots in the line inspection field. Many scholars and research institutes at home or abroad are proceeding with the relevant research on the inspection robot [5 9], but the research on the humanoid dual-arms inspection robot has not be seen in the relevant references. The model of the humanoid dual-arms inspection robot is established which is shown in Fig.1, and the crossing obstacle process of the robot is shown in Fig.3. The inspection robot will stop when it comes across obstacle, and its left driven wheel will be locked as shown in Fig.3 (a). The robotic centroid is adjusted below the left driven wheel, meanwhile the right arm gets off the transmission line and outstretches to the right of obstacle, and then the right driven wheel is hung on the transmission line and is locked as shown in Fig.3 (b). The robotic centroid is adjusted below the right driven wheel as shown in Fig.3(c), finally, this left arm gets off the transmission line and retracts to the right of obstacle and the left driven wheel is hung on the line as shown in Fig.3 (d). Thus, the crossing obstacle process is finished.

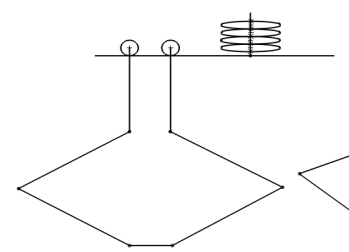

(a)

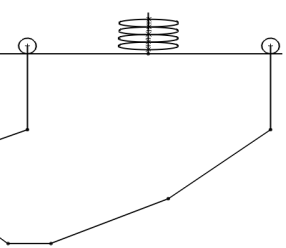

(b)

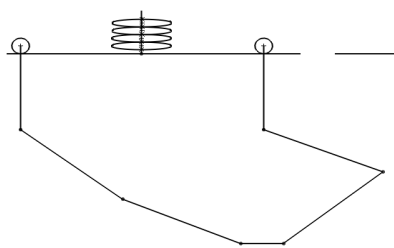

(c)

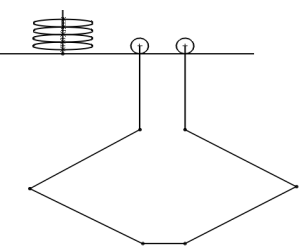

(d)

Fig. 3. Crossing obstacle of the robot

When the inspection robot crosses the obstacle, the elbow joint will bear great torque while the robotic centroid is adjusted to the single arm, which causes the structure of elbow joint and motor power to become larger and influences the dynamic performance and flexibility of the robot. Therefore, in order to reduce the torque, power and weight of the driven motor of the elbow joint, a flexible-cable is installed between the wrist joint and the end of upper arm, which can parallel balance the robot with the elbow joint as shown in Fig.2. When the robotic centroid is adjusted to the single arm, the flexible-cable can mainly balance the gravity of the robot.

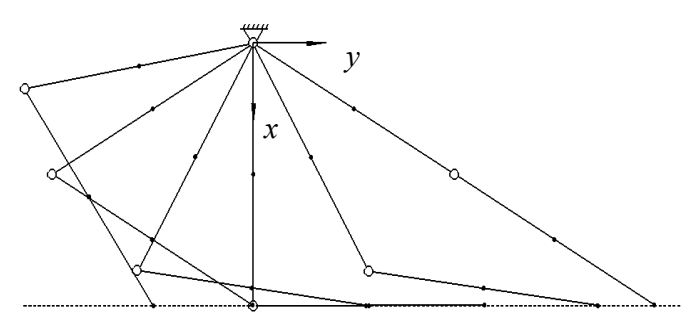

(a) Horizontal movement

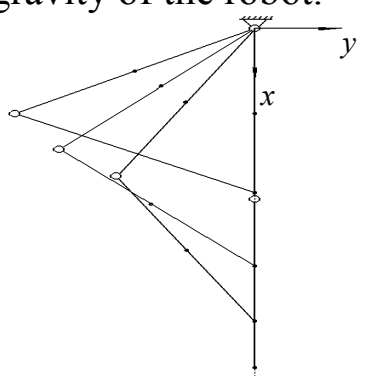

(b) Vertical movement

Fig. 4. Robot movement pattern

The work patterns of the robot include hanging on line, walking, crossing obstacle, getting off line. The robotic pose keeps unchanged in the walking pattern, and the robot moves only horizontally in the crossing obstacle pattern as shown in Fig.4 (a). However, the robot moves only vertically when the robot is working in the hanging on line and getting off line pattern as shown in Fig.4 (b). Therefore, the following dynamics analysis of the robotic arm is based on the horizontal and vertical directions. 


\section{Dynamic Equation of the Serial Driven Robotic Arm}

Force Analysis of the Arm's Joint. In order to study the influence of the flexible-cable to the torque of the wrist and elbow joints, the force situation of the wrist and elbow joints without the flexible-cable is considered firstly. As shown in Fig.3 (b), it is a limit position of the robot in crossing obstacle process, while the left driven wheel is locked and the forearm can be regarded as connecting the fixture through the wrist joint. The torque of the wrist and elbow joints drives the robot to move right. Because the influence of the flexible-cable to the torque of the wrist and elbow joints is the mainly studied objection in this paper, the left arm can be just regarded as the research objection. When the robot adjusts the centroid from the pose shown in Fig.3 (b) to the pose shown in Fig.3(c), two arms bear force, but the left arm bears the most force in the initial stage of adjustment. In order to simplify the problem, the quality of the robot will be equivalent to the end of the upper arm. The force model of the robotic arm is shown in Fig.5 (a) when the robot moves horizontally, and the force model of the robotic arm is shown in Fig.5 (b) when the robot moves vertically.

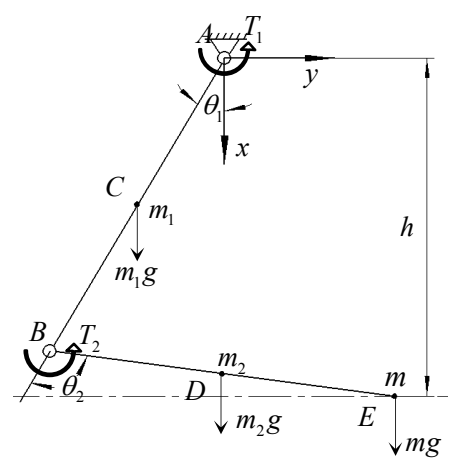

(a) Horizontal movement

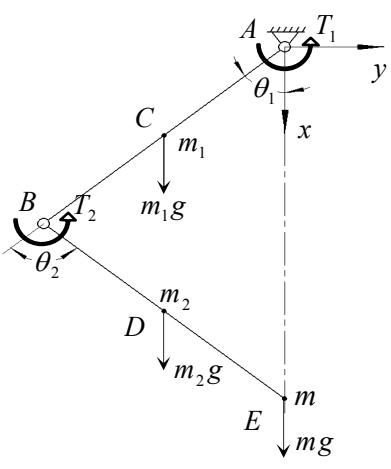

(b) Vertical movement

Fig. 5. Serial driven without the flexible-cable

The upper arm and forearm are both regarded as rigid poles. The respective quality of each pole is $m_{1}=2 \mathrm{~kg}$ and $m_{2}=2 \mathrm{~kg}$. The respective length of each pole is $l_{1}=300 \mathrm{~mm}$ and $l_{2}=300 \mathrm{~mm}$. The robotic concentrated quality is $m=15 \mathrm{~kg}$. The respective torque of the wrist and elbow joint is $T_{1}$ and $T_{2}$, which balances the robot gravity and drives robot to move horizontally and vertically.

Dynamic Equation of the Arm. The coordinate system of the arm is created on the wrist joint, and the parameters $\theta_{1}$ and $\theta_{2}$ are selected for generalized coordinates as shown in Fig.5. According to the speed and coordinate of each centroid point, the total kinetic energy and the total potential energy of the robotic arm can be calculated. Taking them to the Lagrange dynamic equation, the torque of the wrist and elbow joints can be obtained as Eq.1 and Eq.2.

$$
\begin{aligned}
T_{1}= & \frac{d}{d t} \frac{\partial L}{\partial \dot{\theta}_{1}}-\frac{\partial L}{\partial \theta_{1}} \\
= & {\left[\left(\frac{1}{3} m_{1}+m_{2}+m\right) l_{1}^{2}+\left(\frac{1}{3} m_{2}+m\right) l_{2}^{2}+\left(m_{2}+2 m\right) l_{1} l_{2} \sin \theta_{2}\right] \ddot{\theta}_{1}+\left[\left(\frac{1}{3} m_{2}+m\right) l_{2}^{2}+\left(\frac{1}{2} m_{2}+m\right) l_{1} l_{2} \cos \theta_{2}\right] \ddot{\theta}_{2} } \\
& -\left[\left(m_{2}+2 m\right) l_{1} l_{2} \sin \theta_{2}\right] \dot{\theta}_{1} \dot{\theta}_{2}-\left[\frac{1}{2}\left(m_{2}+m\right) l_{1} l_{2} \sin \theta_{2}\right] \dot{\theta}_{2}^{2}-\left(\frac{1}{2} m_{1}+m_{2}+m\right) g l_{1} \sin \theta_{1} \\
& -\left(\frac{1}{2} m_{2}+m\right) g l_{2} \sin \left(\theta_{1}+\theta_{2}\right) \\
T_{2}= & \frac{d}{d t} \frac{\partial L}{\partial \dot{\theta}_{2}}-\frac{\partial L}{\partial \theta_{2}} \\
= & {\left[\left(\frac{1}{3} m_{2}+m\right) l_{2}^{2}+\left(\frac{1}{2} m_{2}+m\right) l_{1} l_{2} \cos \theta_{2}\right] \ddot{\theta}_{1}+\left[\left(\frac{1}{3} m_{2}+m\right) l_{2}^{2}\right] \ddot{\theta}_{2}+\left[\frac{1}{2}\left(m_{2}+m\right) l_{1} l_{2} \sin \theta_{2}\right] \dot{\theta}_{1}^{2} } \\
& -\left(\frac{1}{2} m_{2}+m\right) g l_{2} \sin \left(\theta_{1}+\theta_{2}\right)
\end{aligned}
$$




\section{Dynamic Equation of the Series-Parallel Driven Joint with Flexible-Cable}

Force Analysis of Series-Parallel Joint. In order to reduce the torque of the elbow joint, using the characteristics of the flexible-cable driven mode such as small inertial, high bearing capacity, a flexible-cable connects the wrist joint with the end of upper arm, which will become parallel driven with the elbow joint. So a kind of new-type serial-parallel combination driven joint that combine parallel flexible-cable driven mode in serial joint is designed [10]. The force model of the robotic arm is shown in Fig.6 (a) when the robot moves horizontally, and the force model of the robotic arm is shown in Fig.6 (b) when the robot moves vertically.

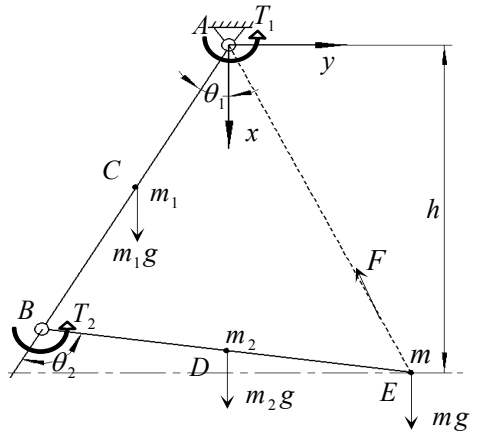

(a) Horizontal movement

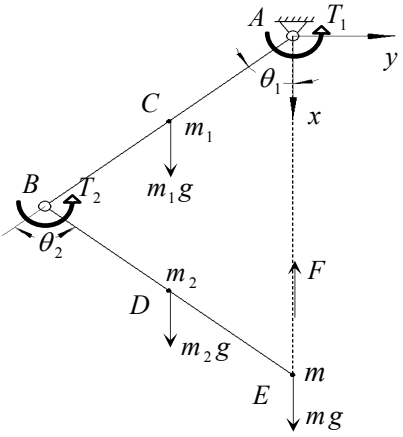

(b) Vertical movement

Fig. 6. Serial-parallel driven with the flexible-cable

The wrist joint connect the elbow joint in series, and they constitute parallel driven with the flexible-cable. The force $(F)$ of the flexible-cable and the torque $\left(T_{1}, T_{2}\right)$ of the wrist and elbow joints commonly balance the gravity of the robot.

Dynamic Equation of the Series-Parallel Joint. Comparing with the quality of the robot, the quality of flexible-cable is very small, so the quality of flexible-cable can be ignored. So that the total kinetic energy and the total potential energy of arm remains unchanged. Supposing the flexible-cable provides a constant force $F=m g$, because the direction of $F$ goes through the axis of the wrist joint, it does not influence the torque $\left(T_{1}\right)$ of wrist, but it will have great influence on the torque $\left(T_{2}\right)$ of the elbow joint. According to the influence of the flexible-cable force on the torque of the wrist and elbow joint, the torque $\left(T_{1}^{\prime}, T_{2}^{\prime}\right)$ of the wrist and elbow joints in the serial-parallel joint with flexible-cable can be given as Eq.3 and Eq.4.

$$
\begin{aligned}
T_{1}^{\prime} & =\frac{d}{d t} \frac{\partial L}{\partial \dot{\theta}_{1}}-\frac{\partial L}{\partial \theta_{1}} \\
= & {\left[\left(\frac{1}{3} m_{1}+m_{2}+m\right) l_{1}^{2}+\left(\frac{1}{3} m_{2}+m\right) l_{2}^{2}+\left(m_{2}+2 m\right) l_{1} l_{2} \sin \theta_{2}\right] \ddot{\theta}_{1}+\left[\left(\frac{1}{3} m_{2}+m\right) l_{2}^{2}+\left(\frac{1}{2} m_{2}+m\right) l_{1} l_{2} \cos \theta_{2}\right] \ddot{\theta}_{2} } \\
& -\left[\left(m_{2}+2 m\right) l_{1} l_{2} \sin \theta_{2}\right] \dot{\theta}_{1} \dot{\theta}_{2}-\left[\frac{1}{2}\left(m_{2}+m\right) l_{1} l_{2} \sin \theta_{2}\right] \dot{\theta}_{2}^{2}-\left(\frac{1}{2} m_{1}+m_{2}+m\right) g l_{1} \sin \theta_{1} \\
& -\left(\frac{1}{2} m_{2}+m\right) g l_{2} \sin \left(\theta_{1}+\theta_{2}\right) \\
T_{2}^{\prime}= & \frac{d}{d t} \frac{\partial L}{\partial \dot{\theta}_{2}}-\frac{\partial L}{\partial \theta_{2}}-F \sin \frac{1}{2} \theta_{2} \sin \left(\pi-\theta_{2}\right) \\
= & {\left[\left(\frac{1}{3} m_{2}+m\right) l_{2}^{2}+\left(\frac{1}{2} m_{2}+m\right) l_{1} l_{2} \cos \theta_{2}\right] \ddot{\theta}_{1}+\left[\left(\frac{1}{3} m_{2}+m\right) l_{2}^{2}\right] \ddot{\theta}_{2}+\left[\frac{1}{2}\left(m_{2}+m\right) l_{1} l_{2} \sin \theta_{2}\right] \dot{\theta}_{1}^{2} } \\
& -\left(\frac{1}{2} m_{2}+m\right) g l_{2} \sin \left(\theta_{1}+\theta_{2}\right)-F \sin \frac{1}{2} \theta_{2} \sin \left(\pi-\theta_{2}\right)
\end{aligned}
$$




\section{Dynamic Simulation Experiment}

Simulation of the Robotic Horizontal Movement. When the robot crosses obstacle and adjusts the centroid to the right as shown in Fig.3 (b) and (c), the robot moves only horizontally not vertically, therefore the value of $h$ keep $h=300 \mathrm{~mm}$ unchanged. So, there is the following restriction equation.

$$
x_{E}=l_{1} \cos \theta_{1}+l_{2} \cos \left(\theta_{1}+\theta_{2}\right)=h
$$

When the robot adjusts the centroid from the left to the right, two arms bear force, but the left arm bears the most force in the initial stage of adjustment. In order to really simulate the force of the wrist and elbow joints, the rotation angle $\left(\theta_{1}\right)$ of the wrist joint should not be too big. Given the wrist joint angular velocity $\left(\omega_{1}=\pi / 18 \mathrm{rad} / \mathrm{s}\right)$ and the simulation time $(t=6 \mathrm{~s})$, the rotation angle $\left(\theta_{1}\right)$ will turn from $-80^{\circ}$ to $-20^{\circ}$. Considering the constraint Eq.5, the $T_{1}, T_{2}, T_{1}^{\prime}, T_{2}^{\prime}$ can be solved and the simulation curves can be gained as shown in Fig.7.

Simulation of the Robotic Vertical Movement. When the robot hangs on line or gets off line, the robot moves only vertically not horizontally, therefore the coordinate of $y_{E}$ keep $y_{E}=0 \mathrm{~mm}$ unchanged. So, there is the following restriction equation.

$$
y_{E}=l_{1} \sin \theta_{1}+l_{2} \sin \left(\theta_{1}+\theta_{2}\right)=0
$$

Given the wrist joint angular velocity $\left(\omega_{1}=\pi / 18 \mathrm{rad} / \mathrm{s}\right)$ and the simulation time $(t=6 \mathrm{~s})$, the rotation angle $\left(\theta_{1}\right)$ will turn from $-80^{\circ}$ to $-20^{\circ}$. Considering the constraint Eq.6, the $T_{1}, T_{2}, T_{1}^{\prime}$, $T_{2}^{\prime}$ can be solved and the simulation curves can be gained as shown in Fig.8.

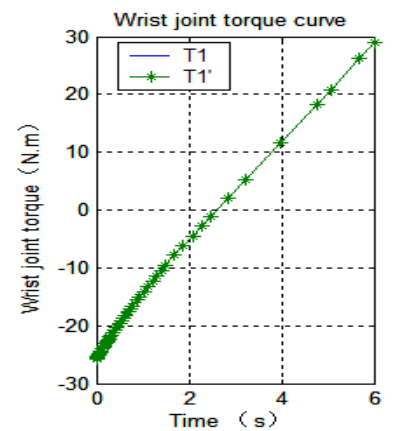

Fig. 7. Simulation result of the robot horizontal movement
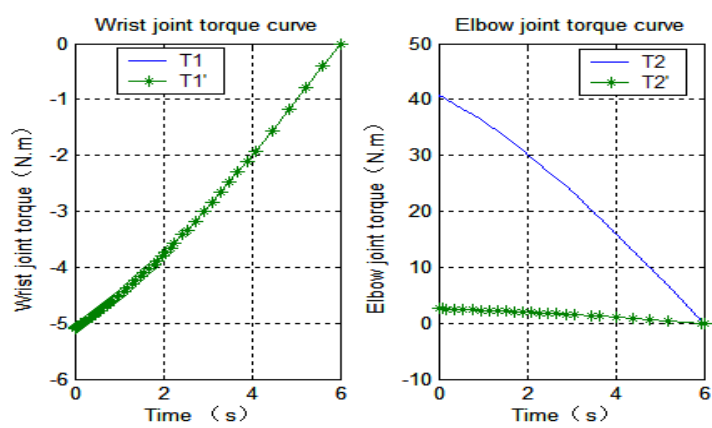

Fig. 8 Simulation result of the robot vertical movement

Analysis of the simulation results. By the simulation results as shown in Fig.7 and Fig. 8 , it can be seen that the curve of $T_{1}$ coincides with the curve of $T_{1}^{\prime}$ and the curve of $T_{2}$ does not coincide with the curve of $T_{2}^{\prime}$. That the curve of $T_{1}$ coincides with the curve of $T_{1}^{\prime}$ shows that the force $F$ of the flexible-cable does not influence the torque of the wrist joint. That the curve of $T_{2}$ does not coincide with the curve of $T_{2}^{\prime}$ shows that the force $F$ of the flexible-cable influences the torque of the elbow joint.

As shown in Fig.7, when the robot moves horizontally, the torque of the wrist joint varies greatly and the max is about $30 \mathrm{Nm}$, however, the max torque of the elbow joint which is influenced by the force of the flexible-cable from $47 \mathrm{Nm}$ reduces to $12 \mathrm{Nm}$.

As shown in Fig.8, when the robot moves vertically, the torque of the wrist joint varies little and the max is about $5 \mathrm{Nm}$, however, the max torque of the elbow joint which is influenced by the force of the flexible-cable from $41 \mathrm{Nm}$ reduces to $3 \mathrm{Nm}$. 


\section{Conclusion and the Future Work}

By analyzing the results of the simulation experiment, it can be seen that comparing with the serial driven joint this serial-parallel combination driven joint with flexible-cable can reduce the elbow torque greatly and improve the load capacity of robotic arm, which can solve the problem that the torque of serial driven joint in the dual-arms inspection robot is too high and provide a new idea for designing the dual-arms transmission line inspection robot. At the same time, it can be seen that this serial-parallel combination driven joint also has some problems. Firstly, it doesn't help for reducing the wrist joint torque, and the wrist joint still bear high torque. Secondly, it is unsatisfied with the reduction of the elbow joint torque and the elbow joint torque is still considerable fluctuation. Thirdly, the tension of the flexible-cable is constant during the dynamic analysis, and the dynamic characteristic of the wrist and elbow joint may be better if the force of the flexible-cable can vary. Therefore, it is necessary to make further researches on the structure design of the robotic arm, the installation position of the flexible-cable and the driven-mode of the flexible-cable.

This work was supported by National Natural Science Foundation of China (project 60875082) and the Fundamental Research Funds for the Central Universities (N090403003).

\section{References}

[1] YUN Yuan, XU Qingsong, LI Yangmin, Survey on Parallel Manipulators with Micro/Nano Manipulation Technology and Applications, CHINESE JOURNAL OF MECHANICAL ENGINEERING. Commun. 12 (2008)12-23.

[2] GAO Yan, LIU Yanwu, YOU Bo, A Study on Avoiding Obstacle for a New Type of Series-Parallel Mechanism, JOURNAL HARBIN UNIV. SCI.\&TECH. Commun. 2(2001)41-45.

[3] WANG Wei, ZHANG Houxiang, DENG Zhicheng, Reconfigurable Mobile Robot Based on Serial and Parallel Mechanism, CHINESE JOURNAL OF MECHANICAL ENGINEERING. Commun. 5 (2008)92-101.

[4] Yu Shunnian, Ma Luzhong, Chen Exi, Study on a Novel Series-parallel Chinese Massage Manipulator, China Mechanical Engineering. Commun. 10 (2005)1773-1778.

[5] Gongping $\mathrm{Wu}$, Xianjin Xu, Hua Xiao, A Novel Self-navigated Inspection Robot along High-Voltage Power Transmission Line and Its Application, ICIRA 2008, Part II, Berlin, 2008, pp.1145-1154.

[6] Ludan Wang, Fei Liu, Shaoqiang Xu, Design and Analysis of a Line-walking Robot for Power Transmission Line Inspection, Proceedings of the 2010 IEEE International Conference on Information and Automation, Harbin, 2010, pp. 1398-1403.

[7] Nicolas Pouliot and Serge Montambault, Hydro-Québec LineScout Technology: From Inspection to Robotic Maintenance on Live Transmission Power Lines, 2009 IEEE International Conference on Robotics and Automation Kobe International Conference Center Kobe, Japan, 2009, pp.1034-1040.

[8] L.J.Fang and H.G.Wang, Research on the Motion System of the Inspection Robot for 500kV Power Transmission Lines, 2010 1st International Conference on Applied Robotics for the Power Industry, Canada, 2010.

[9] Jaka Katrasnik, Franjo Pernus, and Bostjan Likar, A Survey of Mobile Robots for Distribution Power Line Inspection, IEEE TRANSACTIONS ON POWER DELIVERY. Commun. 25(2010)485-493.

[10] LIU Jie, NING Ke-jun, ZHAO Ming-yang, Model of a New Type Wire Driven Parallel Robot System, Journal of Northeastern University. Commun. 10 (2002)988-991. 